\title{
Identification of Candidate Cancer Predisposing Genes by Whole-Exome Sequencing of Families at High-Risk of Glioma
}

\author{
Ben Kinnersley 1* ${ }^{*}$, Peter Broderick ${ }^{1}$, Daniel Chubb ${ }^{1}$, Hanne Bødtcher ${ }^{2}$, Karim Labreche ${ }^{3}$, Axel de Bernardi ${ }^{3}$, Oliv- \\ ier Chinot ${ }^{4}$, Christoffer Johansen 5, Marc Sanson ${ }^{3,6}$ and Richard Houlston ${ }^{1}$,
}

1 Division of Genetics and Epidemiology, The Institute of Cancer Research, Sutton, London, SM2 5NG, UK; ben.kinnersley@icr.ac.uk (B.K.); peter.broderick@icr.ac.uk (P.B.); daniel.chubb@icr.ac.uk (D.C.); richard.houlston@icr.ac.uk (R.H.)

2 Danish Cancer Society Research Center, Strandboulevarden 49, DK-2100 Copenhagen, Denmark; hab@cancer.dk (H.B.)

3 Sorbonne Université, Inserm, CNRS, UMR S 1127, Paris Brain Institute - Institut du Cerveau (ICM), Paris, France. Equipe labellisée LNCC; karim.labreche@icm-institute.org (K.L.); axel.debernardi@icm-institute.org (A.D-B.); marc.sanson@aphp.fr (M.S.)

4 Aix-Marseille Univ, APHM, CNRS, INP, Inst Neurophysiopathol, La Timone Hospital, Neurooncology Department, Marseille, France; olivier.chinot@ap-hm.fr (O.C.)

5 Late effects Research, CASTLE 9601, Department of Oncology, Rigshospitalet, University of Copenhagen, Blegdamsvej 58, 2100 Copenhagen, Denmark; christoffer.johansen@regionh.dk (C.J.)

6 AP-HP, Groupe Hospitalier Pitié-Salpêtrière, Service de Neurologie 2-Mazarin, Paris, France

* Correspondence: ben.kinnersley@icr.ac.uk; Tel.: +442034376194

\begin{abstract}
Simple Summary: As with many cancers, a person's risk of glioma is approximately twice that compared to the general population if they have a family member affected by glioma. This suggests that there is a substantial inherited component of the familial risk of glioma, but which exact genes are involved are largely unknown. To further investigate this, we sequenced 17 glioma families to prioritise inherited gene mutations shared in multiple glioma patients in the same family. We compared against genetic mutations identified from sequencing of 150 unrelated familial glioma patients as well as publicly available data from The Cancer Genome Atlas (TCGA) of 691 glioma patients. We identified a number of novel potential glioma predisposition genes which require further investigation to more fully understand their contribution to glioma risk.
\end{abstract}

\begin{abstract}
The genetic aetiology of familial glioma is largely unknown. To gain further insight into the role of rare disruptive variants we performed whole exome sequencing (WES) of 17 glioma families and 150 unrelated familial glioma cases, additionally leveraging WES data on 691 glioma cases from The Cancer Genome Atlas (TCGA). We. identify two families where loss of function mutations in dynein axonemal heavy chain 11 (DNAH11) co-segregated with glioma (DNAH11 p.Ser1470ArgfsTer6 and p.Thr3900Lys) and single families implicating Telomere Replication Complex Component 1 (CTC1 p.Leu1058ProfsTer32) and filamin alpha (FLNA p.Ser1356Phe). Our analysis does not provide support for a hitherto unidentified major cancer gene for glioma but highlights a series of novel candidate predisposition variants and genes, worthy of further investigation to shed light on glioma risk.
\end{abstract}

Keywords: glioma; genetics; inherited; mutation; germline

\section{Introduction}

Gliomas account for $40 \%$ of all primary malignant brain tumours (PBT) and are responsible for around 13,000 cancer-related deaths in the US annually [1]. Despite advances in surgery, radiotherapy and chemotherapy the prognosis for most gliomas remains poor with the most common type of glioma, glioblastoma (GBM), typically having a median survival of only 14 months [2]. 
Family history of brain tumours is an important risk factor in a small proportion of patients with primary glial neoplasms of the central nervous system. Among these patients, a subset carry pathogenic germline alterations in tumour suppressor genes as part of well-characterized tumour predisposition syndromes [2,3]. These include mutations in NF1 as part of neurofibromatosis type 1 syndrome, TP53 as part of Li-Fraumeni syndrome (OMIM 151623), mismatch repair genes (e.g. MLH1, MSH2, MSH6, PMS2) as part of Lynch/Turcot syndrome (MIM 276300) and CDKN2A responsible for familial melanomaastrocytoma syndrome (MIM 155755). Each of these disorders are rare and even collectively, they only account for little of the two-fold increased risk of glioma observed in relatives of patients [4].

Genome-wide association studies have demonstrated that common single-nucleotide polymorphisms influence glioma risk [5-10], affirming a complex underlying architecture for its genetic aetiology but one that appears weighted disproportionately toward rare disease-causing variants. This class of genetic susceptibility is mechanistically important and highly relevant to the clinical management of glioma patients. Support for the existence of additional hitherto unidentified, rare susceptibility alleles for glial tumours has been provided by studies linking POT1 (Protection of telomeres 1) mutations with glioma development [11] in addition to risk of melanoma [12] and leukaemia [13]. POLE as a cancer susceptibility gene (CSG) was first described in the context of dominantly inherited colorectal cancer [14] and led to the syndrome of polymerase proof reading polyposis (PPRP) being recognised. Reports of further PPRP families has shown that the spectrum of cancer risk associated with POLE mutations extends to include other tumour types including glioma [15].

To gain further insight into the role of rare disruptive variants in glioma we performed whole exome sequencing (WES) of germline DNA from 150 glioma families to identify genes containing disease-segregating variants. We performed complementary statistical approaches to identify different classes of disease-causing variants using these data and WES data on 691 glioma cases from The Cancer Genome Atlas Program (TCGA). Briefly, the exomes of 168 glioma cases were examined for genetic variants segregating in 17 families where multiple cases were available for sequencing, searching for additional support in TCGA exome data. Candidate predisposition genes were then followed-up by validation sequencing of segregating variants within families. Finally, to assess for a burden of variants across a gene a combined set of 841 cases (150 familial glioma and 691 TCGA) were tested against 1,609 controls.

\section{Materials and Methods}

\subsection{Familial glioma cases}

Glioma cases enriched for genetic susceptibility were recruited through two sources - the Service de Neurologie Mazarin, Groupe Hospitalier Pitié-Salpêtrière Paris and Danish cancer centres that had participated in the Gliogene consortium. Collectively, this provided for an ascertainment of 169 glioma cases from 150 families with multiple cases of primary brain tumours and 13 cases with a personal history of both glioma and melanoma. All index cases had histologically proven glioma and tumour diagnoses in relatives established through medical records and/or cancer registrations. None of the index cases had a family history of classical Li-Fraumeni syndrome or the clinical features meeting internationally recognised criteria for a diagnosis of neurofibromatosis [16]. Germline DNA was extracted from EDTA-peripheral blood samples by standard methods and quantified using PicroGreen (Invitogen). All subjects gave written informed consent and the study had ethical approved by research committees at respective centres. Supplementary Table 1 provides the clinico-patholgical details of the 150 families studied.

\subsection{Whole exome sequencing of glioma cases}


Whole exome sequencing of germline DNA was performed using Illumina's Nextera Rapid Capture Exome Enrichment Kit in conjunction with Illumina HiSeq 2000 technology (Illumina, San Diego, CA). Paired-end FASTQ files of $100 \mathrm{bp}$ reads were extracted using CASAVA software (version 1.8.1; Illumina) and aligned to build 37 (hg19) of the human reference genome using Stampy v1.0.23 (http://www.well.ox.ac.uk/project-stampy) [17] and Burrows-Wheeler Aligner v0.5.9 (http://bio-bwa.sourceforge.net/) [18] software and the Genome Analysis Tool Kit (GATK) pipeline (version 3.2-2), according to best practice $[19,20]$. Across the $37 \mathrm{Mb}$ Nextera capture region, the median coverage across on 168 samples was $62 \mathrm{X}$, with median $91.6 \%$ of bases covered at $>15 \mathrm{X}$. Variants were filtered for positions found in $>1$ sample from 1,609 UK 1958 Birth Cohort (BC) samples [21], which had been sequenced in-house using the same Illumina methodology (EGAD00001001021). We also filtered variants based on frequencies in the 1000 Genomes Project, National Heart, Lung, and Blood Institute Exome Sequencing Project (ESP6500), and the Exome Aggregation Consortium (ExAC) catalog. We used principal component analysis of SNP genotypes to examine population substructure of cases restricting downstream analyse to individuals with more than $80 \%$ European ancestry (Supplementary Fig. 1). Following GATK variant quality score recalibration (VQSR), variants were excluded if in the $99.5 \%$ tranche or above for SNVs and in the 99\% tranche or above for INDELs. Genotypes were retained if GQ (genotype quality) > 30, AD (alternate depth) for alternate alleles $>3$ and DP (total depth) $>6$. Variant annotations for canonical ensembl v78 transcripts were obtained from the Variant Effect Predictor (VEP) [22], taking the gene with the worst possible predicted consequence if a variant overlaps multiple genes. VEP annotations and functional classifications from CONDEL were used to predict the impact of variants [23].

\subsection{Processing of TCGA glioma exomes}

Germline (blood) and tumour exome bam files for 752 glioma cases from TCGA were downloaded (444 low-grade glioma (LGG) and 308 GBM) (accession number phs000178.v11.p8). Across the 37Mb Nextera capture region, the median coverage across all samples was $79 \mathrm{X}$, with a median of $83.4 \%$ of bases covered $>15 \mathrm{X}$. Base call qualities were reverted to raw qualities and reads shuffled prior to realignment to human build 37, with samples processed along the exome analysis pipeline as above. Reported tumour histology was revised to reflect the revised WHO v5 classification [24], making reference to molecular information from Ceccarelli et al., 2016 [25]. Specifically: "Oligoastrocytoma" was revised to "Oligodendroglioma" if there was evidence of $1 p / 19 q$ co-deletion otherwise histology was revised to "Astrocytoma"; "GBM" was revised to "Astrocytoma" if there was evidence of IDH mutation; "Astrocytoma" was revised to "GBM" if there was no IDH mutation and at least one of the following molecular criteria were met (TERT promoter mutation, combined chr7 gain and chr10 loss, EGFR amplification).

\subsection{Bioinformatics analyses}

Glioma cases were evaluated for pathogenic mutations in Cancer Susceptibility Genes (CSGs) which have established roles in glioma predisposition (i.e. mono-allelic TP53, NF1, NF2, CDKN2A/p16, and bi-allelic mismatch repair gene mutations). Variants were considered as pathogenic if exhibiting ExAC MAF $<0.5 \%$ and a "pathogenic" or "likely pathogenic" NCBI ClinVar annotation.

To ensure only rare variants were retained for the segregation analysis, variants were excluded if the observed allele count (AC) was $>1$ in any of 1000 Genomes Project v3 ( $n=2,504$ individuals), Exome variant server $(n=6,503)$, UK10K $(n=3,897)$ or 1958-BC $(n=1,609)$ or if the AC was $>100$ in $\operatorname{ExAC}(n=60,706)$. Positions resulting in protein-altering changes were identified using Ensembl VEP (version 78) and variants shared between family members annotated using custom scripts. Cases with multiple variants in the same gene were manually examined using the Interactive Genomics Browser (IGV) to establish haplotypes. For the segregation analysis variants were restricted to those annotated as 


\begin{abstract}
"high impact" (i.e. protein truncating - nonsense, frameshift, splice acceptor/donor variants) by the variant effect predictor, as well as missense variants that were annotated as deleterious by CONDEL [23].

Loss-of-heterozygosity (LOH) in TCGA tumours was performed using ExomeCNV [26]. Evidence of allelic imbalance within a $1 \mathrm{Mb}$ window of a given query gene was used to assign LOH status. For gene burden testing, within each glioma family that contained multiple affected samples, the sample with the best coverage was retained. Statistical association testing was performed in PLINK/SEQ v0.09 (https://zzz.bwh.harvard.edu/plinkseq/) annotating variants to ensemble v78 transcripts. All previously mentioned variant and genotype filters were applied, additionally only variants with $<5 \%$ null genotypes were retained. For gene-based tests SKAT-OPTIMAL (SKAT-O) was employed which carries out a SKAT and burden test and reports the minimum $P$-value after correcting for multiple testing [27]. Per-gene association $P$-values were calculated restricting to protein-altering variants with $\mathrm{MAF}<0.05$. An additional test was conducted restricted to synonymous variants in each gene, as an indicator of case-control biases e.g. unequal coverage.
\end{abstract}

\title{
3. Results
}

3.1. Whole exome sequencing and variants in known glioma susceptibility genes

Of the 182 familial glioma cases subjected to WES, data on 10 cases were excluded because of either poor coverage $(n=6)$ or an abnormally high heterozygote count $(n=4)$. Four of the cases were identified as carrying a pathogenic mutations in NF1 $(n=1)$ or TP53 $(n=3)$. This resulted in WES data on 168 cases from 150 families being available for subsequent analyses (Supplementary Table 1). After QC and inspection of PCA plots 59 of the 752 TCGA cases were excluded because of non-European ancestry (Supplementary Fig. 1). Two cases were identified with a germline pathogenic mutation in either CDKN2A or TP53 and these were excluded leaving 691 cases for further analyses.

\subsection{Identification of candidate susceptibility genes}

To maximize the prospects of identifying rare disease-causing variants for glioma, we initially focused our search on the 17 families with multiple affected members with WES data, comprising 35 affected family members (16 families with two affected members, one family with three affected members). After applying population frequency filters we required the filtered variants to be present in all sequenced affected within the family. To further filter the variants identified, we prioritized truncating variants (nonsense, splice acceptor/donor, and frameshift) as well as missense variants predicted to be damaging. For families where multiple genes segregated, genes were prioritised based on the functional impact of the segregating variant. After searching for additional support from 691 TCGA glioma exomes, DNAH11 was the only gene featuring damaging variants segregating in two families. In addition to DNAH11 NEDD4, CTC1 and FLNA were selected for follow-up based on biological plausibility (Fig. 1; Table 1). We confirmed the mutation status for each of these four genes in the respective families by Sanger sequencing, with additional unaffected family members sequenced where available to aid interpretation of variant segregation. 


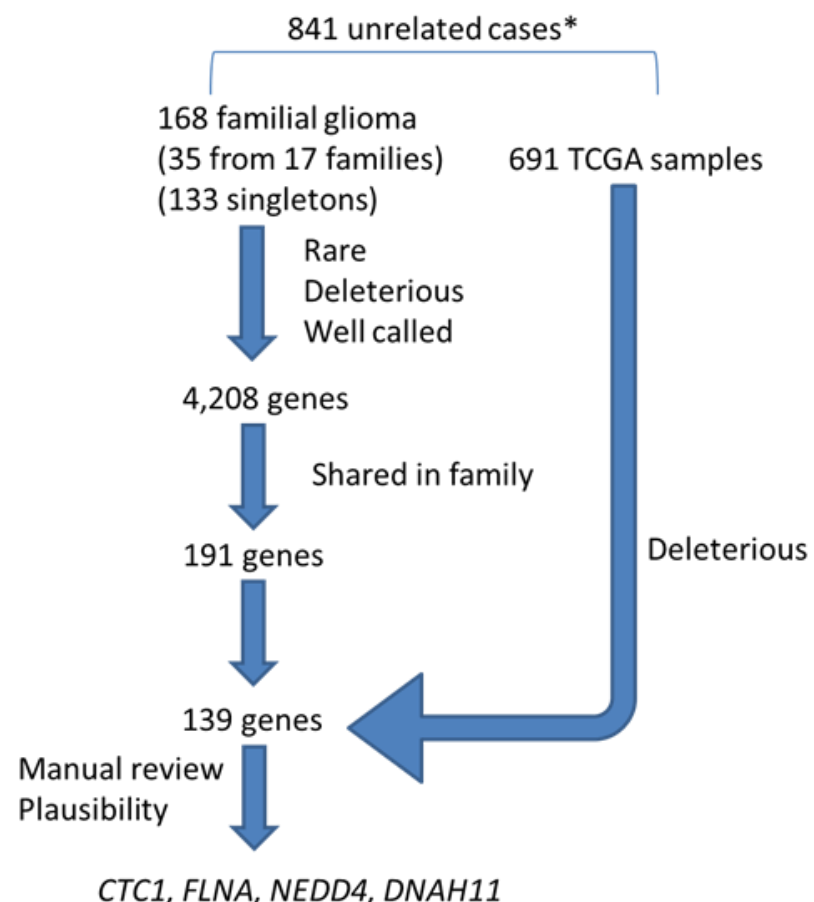

Figure 1. Overview of segregation analysis strategy.

1. Initial variant filtering criteria:

a) Well-called (passing QC threshold);

b) Rare in population;

c) Deleterious annotation (protein truncating variant or missense variant predicted to be deleterious by Condel)

2. Only include genes containing at least one variant shared within a family for the 17 families where multiple individuals were sequenced per family.

3. Only include genes containing at least 1 additional variant in validation series of 691 TCGA glioma and 133 familial glioma exomes.

4. Selection of genes for follow-up based on manual review. Genes were prioritised based on:

a) Biological plausibility;

b) Number of variants shared within family/families;

c) Mode of variant inheritance (based on pedigree and nature of segregating variant). 
Table 1. Damaging protein-altering variants in DNAH11, FLNA, NEDD4 and CTC1. Variant annotations with respect to canonical transcript ENST00000328843.6 and protein ENSP00000330671.6 for DNAH11, ENST00000369850.3 and ENSP00000358866.3 for FLNA, ENST00000315684.8 and ENSP00000313759.8 for CTC1 and ENST00000338963.2 and ENSP00000345530.2 for NEDD4. ${ }^{*}$ Male (X chromosome); ${ }^{1}$ Manual inspection of reads supporting variant call reveals both variants lie on same haplotype.

\begin{tabular}{|c|c|c|c|c|c|c|c|c|c|}
\hline Variant & SNP & Call & Symbol & Transcript change & Protein change & Consequence & $\mathbf{N}$ & Carrier/s & Histology \\
\hline 7:21639426_G/A & & $0 / 1$ & DNAH11 & c. $2689 \mathrm{G}>\mathrm{A}$ & p.Ala897Thr & Missense & 1 & TCGA-32-2616 & GBM \\
\hline $7 \cdot 21641082 \mathrm{~A} / \mathrm{G}$ & $r s 370436662$ & $0 / 1$ & DNAH11 & C $3494 A>G$ & n. Asn $1165 \mathrm{Glv}$ & Missense & 2 & FAM_131 & GBM \\
\hline $1.21041002-\mathrm{H} / \mathrm{G}$ & 15370400002 & 0/1 & DIVRIII & - & 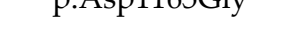 & Avisserise & & TCGA-06-0122 & GBM \\
\hline 7:21659586_ATCAG/A & & $0 / 1$ & DNAH11 & c.4406_4409delTCAG & p.Ser1470ArgfsTer6 & Frameshift & 2 & FAM_7 & Oligodendroglioma, GBM \\
\hline 7:21730450_G/C & & $0 / 1$ & DNAH11 & c. $6013 \mathrm{G}>\mathrm{C}$ & p.Gly2005Arg & Missense & 1 & TCGA-26-1439 & GBM \\
\hline 7:21737767_C/T & rs371460473 & $0 / 1$ & DNAH11 & c. $6137 \mathrm{C}>\mathrm{T}$ & p.Ala2046Val & Missense & 1 & FAM_83 & Astrocytoma \\
\hline 7:21765502_G/C & & $0 / 1$ & DNAH11 & c. $7361 \mathrm{G}>\mathrm{C}$ & p.Gly2454Ala & Missense & 1 & TCGA-12-5299 & GBM \\
\hline 7:21827197_A/G & & $0 / 1$ & DNAH11 & c. $9941 \mathrm{~A}>\mathrm{G}$ & p.Tyr3314Cys & Missense & 1 & TCGA-06-5417 & Astrocytoma \\
\hline 7:21840870_G/C & & $0 / 1$ & DNAH11 & c. $10163 \mathrm{G}>\mathrm{C}$ & p.Arg3388Thr & Missense & 1 & FAM_56 & Gliomatosis cerebri-choroidal \\
\hline 7:21856193_G/A & & $0 / 1$ & DNAH11 & c. $10462 \mathrm{G}>\mathrm{A}$ & p.Glu3488Lys & Missense & 1 & TCGA-06-5418 & GBM \\
\hline 7:21882228_A/T & & $0 / 1$ & DNAH11 & c. $10779 \mathrm{~A}>\mathrm{T}$ & p.Lys3593Asn & Missense & 1 & TCGA-DU-6410 & Oligodendroglioma \\
\hline 7:21893945_A/G & & $0 / 1$ & DNAH11 & c. $11095 \mathrm{~A}>\mathrm{G}$ & p.Lys3699Glu & Missense & 1 & TCGA-CS-6186 & Astrocytoma \\
\hline 7:21893963_A/G & & $0 / 1$ & DNAH11 & c. $11113 \mathrm{~A}>\mathrm{G}$ & p.Ile3705Val & Missense & 1 & TCGA-06-0221 & Astrocytoma \\
\hline 15:56134347_G/C & & $0 / 1$ & NEDD4 & c. $2664 C>G$ & p.Asn888Lys & Missense & 1 & TCGA-HT-7474 & Astrocytoma \\
\hline 15:56139199_A/G & & $0 / 1$ & NEDD4 & c. $2617 \mathrm{~T}>\mathrm{C}$ & p.Tyr873His & Missense & 1 & TCGA-FG-7634 & Oligodendroglioma \\
\hline 15:56155204_G/A & & $0 / 1$ & NEDD4 & c. $1622 \mathrm{C}>\mathrm{T}$ & p.Pro541Leu & Missense & 2 & FAM_2 & Oligoastrocytoma, GBM \\
\hline 15:56208305_T/A & & $0 / 1$ & NEDD4 & c. $725 \mathrm{~A}>\mathrm{T}$ & p.Glu242Val & Missense & 1 & TCGA-28-6450 & GBM \\
\hline X:153581143_G/A & & $1^{*}$ & FLNA & c. $6376 \mathrm{C}>\mathrm{T}$ & p.Pro2126Ser & Missense & 1 & TCGA-FG-8185 & Astrocytoma \\
\hline X:153581377_C/T & & $0 / 1$ & FLNA & c. $6218 \mathrm{G}>\mathrm{A}$ & p.Arg2073His & Missense & 1 & TCGA-DU-7290 & GBM \\
\hline X:153587685_G/A & & $1^{*}$ & FLNA & c. $4232 \mathrm{C}>\mathrm{T}$ & p.Ser1411Leu & Missense & 1 & TCGA-06-2565 & GBM \\
\hline X:153587927_G/A & & $1^{*}$ & FLNA & c. $4067 \mathrm{C}>\mathrm{T}$ & p.Ser1356Phe & Missense & 2 & FAM_5 & GBM, GBM \\
\hline $\mathrm{X}: 153588742 \_\mathrm{C} / \mathrm{T}$ & rs201908251 & $0 / 1$ & FLNA & c. $3421 \mathrm{G}>\mathrm{A}$ & p.Ala1141Thr & Missense & 1 & TCGA-DŪ-8168 & Oligodendroglioma \\
\hline X:153589860_A/G & & $0 / 1$ & FLNA & c. $3023 \mathrm{~T}>\mathrm{C}$ & p.Ile1008Thr & Missense & 1 & FAM_73 & GBM/melanoma \\
\hline
\end{tabular}




\begin{tabular}{|c|c|c|c|c|c|c|c|c|c|}
\hline Variant & SNP & Call & Symbol & Transcript change & Protein change & Consequence & $\mathbf{N}$ & Carrier/s & Histology \\
\hline X:153592709_G/A & & $1^{*}$ & FLNA & c. $2054 \mathrm{C}>\mathrm{T}$ & p.Thr685Ile & Missense & 1 & FAM_63 & Astrocytoma \\
\hline 17:8132101_C/T & rs374284337 & $0 / 1$ & CTC1 & c. $3331 \mathrm{G}>\mathrm{A}$ & p.Val1111Ile & Missense & 1 & TCGA-76-6282 & GBM \\
\hline 17:8132508_A/AG & & $0 / 1$ & CTC1 & c.3172dupC & p.Leu1058ProfsTer32 & Frameshift & 2 & FAM_1 & Astrocytoma, Astrocytoma \\
\hline 17:8133619_C/A & & $0 / 1$ & CTC1 & c. $2926 \mathrm{G}>\mathrm{T}$ & p.Val976Phe & Missense & 1 & TCGA-HT-7902 & Astrocytoma \\
\hline 17:8133629_C/A & & $0 / 1$ & CTC1 & c. $2916 \mathrm{G}>\mathrm{T}$ & p.Leu972Phe & Missense & 1 & TCGA-HT-7902¹ & Astrocytoma \\
\hline 17:8135066_C/T & & $0 / 1$ & CTC1 & c. $2453 \mathrm{G}>\mathrm{A}$ & p.Arg818Gln & Missense & 1 & TCGA-TQ-A7RV & Astrocytoma \\
\hline 17:8135082_G/A & & $0 / 1$ & CTC1 & c. $2437 \mathrm{C}>\mathrm{T}$ & p.Pro813Ser & Missense & 1 & TCGA-HT-7611 & Astrocytoma \\
\hline 17:8138182_C/A & & $0 / 1$ & CTC1 & c. $1502 \mathrm{G}>\mathrm{T}$ & p.Ser501Ile & Missense & 1 & TCGA-HT-7475 & Astrocytoma \\
\hline 17:8139434_G/C & & $0 / 1$ & CTC1 & c. $1019 C>G$ & p.Ser340Trp & Missense & 1 & TCGA-HT-A614 & Astrocytoma \\
\hline 17:8139591_C/T & rs201553464 & $0 / 1$ & CTC1 & c. $862 \mathrm{G}>\mathrm{A}$ & p.Val288Met & Missense & 1 & TCGA-32-4208 & Astrocytoma \\
\hline 17:8140784_C/A & & $0 / 1$ & CTC1 & c.701G $>\mathrm{T}$ & p.Ser234Ile & Missense & 1 & TCGA-DU-A7T8 & Astrocytoma \\
\hline
\end{tabular}


DNAH11 p.Ser1470ArgfsTer6 (7p15.3) was shared by the affected proband and af- 6 fected paternal uncle of FAM_7, and not by the proband's mother, who had a personal history of Meningioma but has no other family history of brain tumours (Fig. 2a).

DNAH11 p.Thr3900Lys shows incomplete penetrance in FAM_3, being carried by three out of four affected family members (including an obligate carrier; Fig. 2b). Sixteen damaging missense variants in DNAH11 were also observed (Table 1).

Segregation of FLNA p.Ser1356Phe (Xq28) was consistent with X-linked inheritance in FAM_5, with two male affected maternal cousins carrying the variant, and three unaffected female heterozygote carriers (Fig. 2c). The additional unaffected male (brother of proband) did not found to carry the variant. There were six additional damaging missense variants in FLNA (three male homozygotes, three female heterozygotes, Table 1).

CTC1 p.Leu1058ProfsTer32 (17p13.1) segregated completely in FAM_1, with both affected brothers carrying the variant, and both unaffected sisters not carrying it (Fig. 2d). Nine additional damaging variants in CTC1 were observed in glioma cases (Table 1).

In FAM_2, NEDD4 p.Pro541Leu (15q21.3) showed incomplete segregation with glioma, with an unaffected carrier (paternal aunt of proband, Fig. 2e). We observed seven additional damaging NEDD4 variants (Table 1). 6 7
8

8

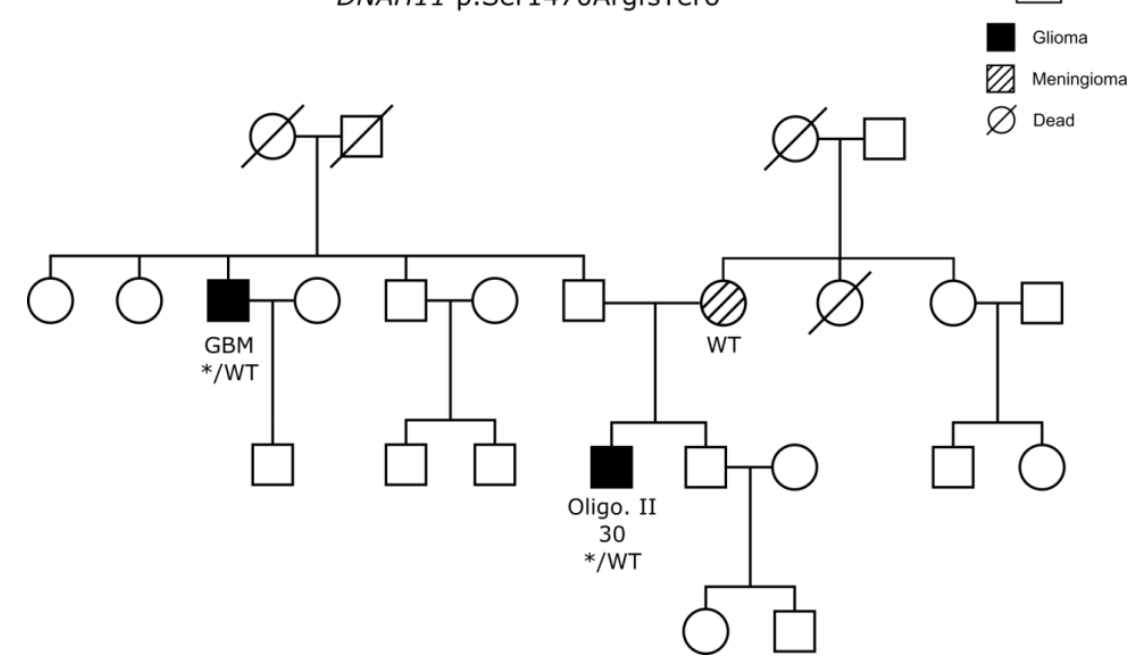

(a)

Segregation of DNAH11 p.Thr3900Lys
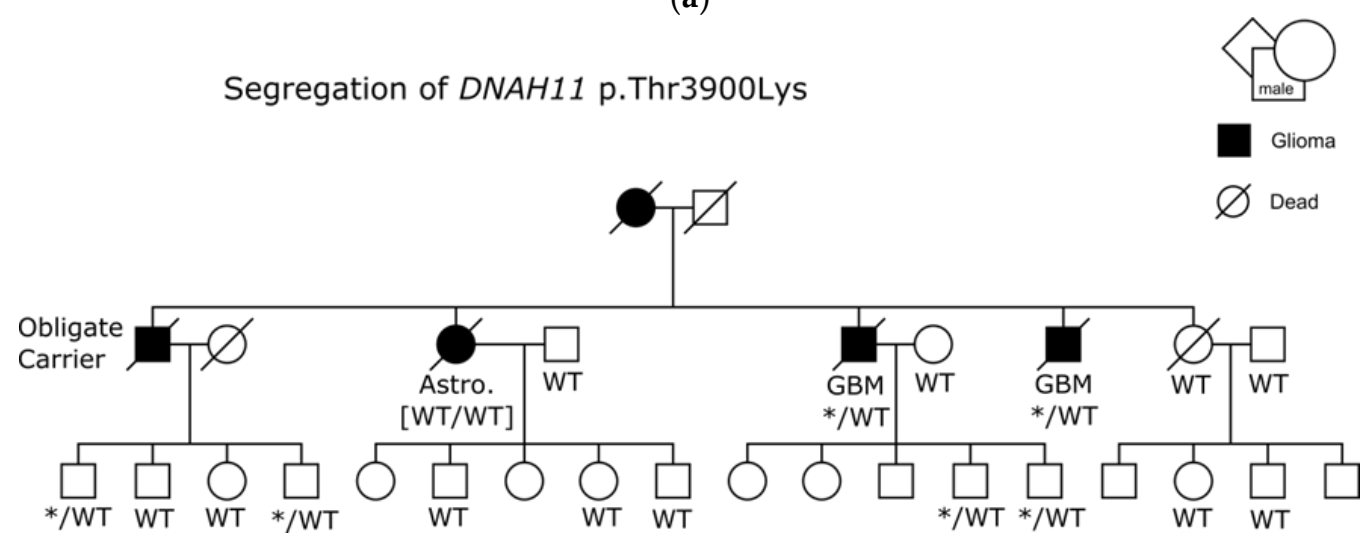

(b) 


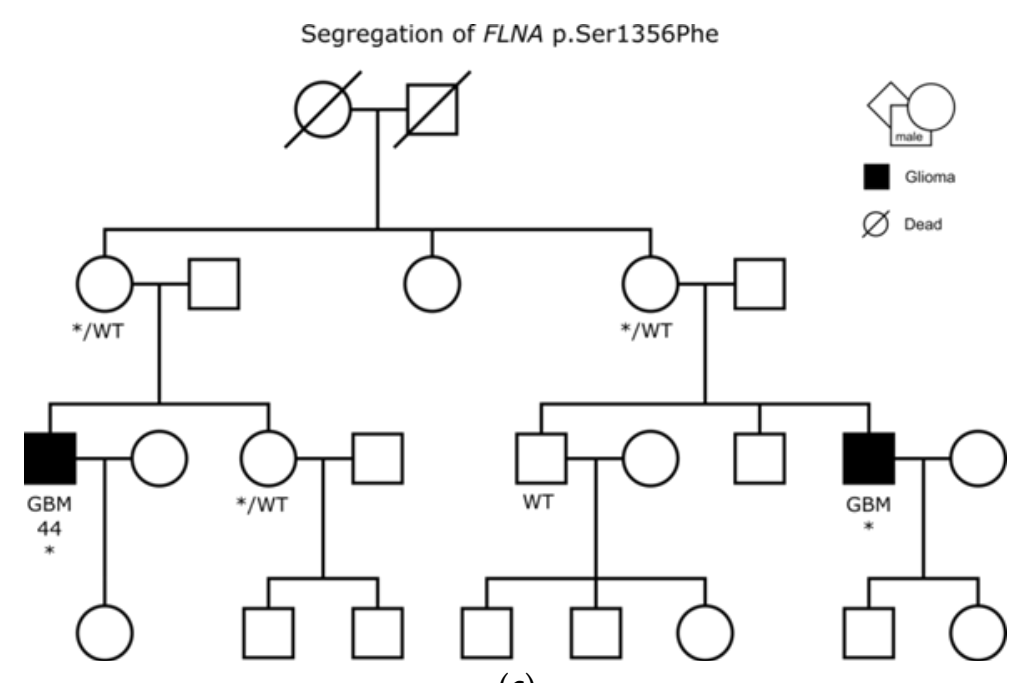

(c)

Segregation of CTC1 p.Leu1058ProfsTer32

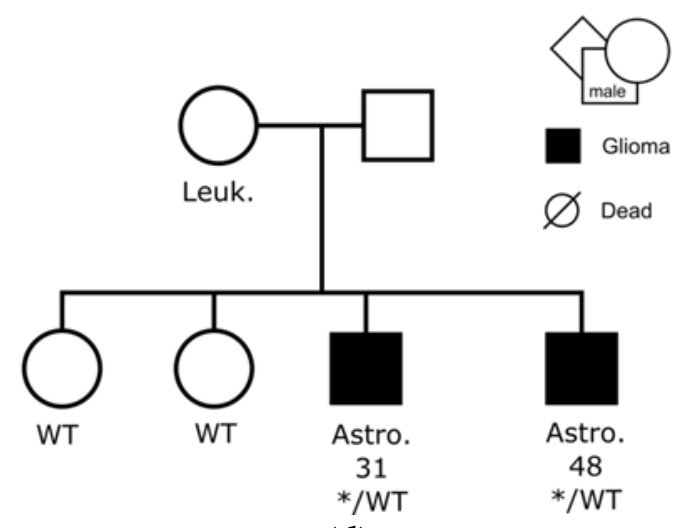

(d)

Segregation of NEDD4 p.Pro541Leu

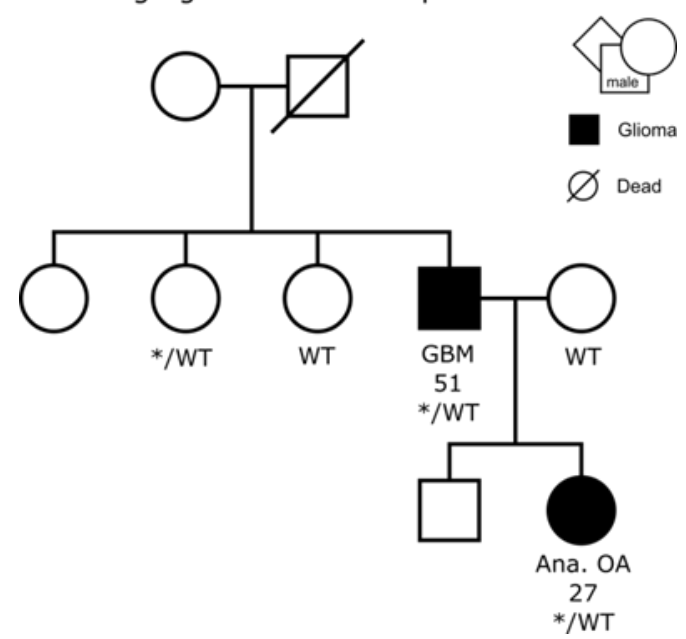

(e)

Figure 2. Segregation of variants in DNAH11, FLNA, CTC1 and NEDD4 in glioma families. [] indicates that a tumour sample was sequenced.

To assess whether germline mutations in these genes are involved in glioma progression, loss-of-heterozygosity ( $\mathrm{LOH}$ ) analysis was carried out in matched Tumour-normal TCGA samples carrying damaging variants in the genes. While there was little evidence for $\mathrm{LOH}$ at NEDD4, DNAH11 or FLNA, four out of nine CTC1 variant carriers showed $\mathrm{LOH}$ at 17p13.1 (Table 2). 


\subsection{Contribution of shelterin complex variants}

Following a similar strategy to that detailed above but without requiring a segregating variant, we examined for damaging mutations in the shelterin genes, POT1, TERF1, TERF2, TINF2, TERF2IP and ACD. In the 150 familial glioma cases only one deleterious missense variant in $A C D$ was identified. Examination of 691 TCGA glioma cases led to the additional identification of eight deleterious shelterin missense variants: two each in POT1 (p.Gly272Arg and p.Arg117Cys) and TERF1 (p.Ala21Ser and p.Thr122Met), and one each in TINF2 (p.Pro430Leu), TERF2IP (p.Arg259Trp) and TERF2 (p.Lys353Glu; Table 3).

\subsection{Gene-burden analysis}

To examine whether any genes exhibited a significant burden of protein-altering variants in cases versus controls SKAT-O tests were carried out on a combined set of 841 cases and 1,609 controls. 131,942 protein-altering variants mapped to 13,076 genes containing $>1$ variant per gene (mean and median variants per gene 10.1 and 7 respectively). No gene showed an association that remained significant after correcting for testing on 13,076 genes $\left(P<3.92 \times 10^{-6}\right)$. Genes with $P$-values $<1 \times 10^{-4}$ are shown in Supplementary Table 2. None of the genes showed a significant association when restriction to synonymous variants, arguing against case-control biases in coverage or calling causing the observed association with protein-altering variants. Of FLNA, NEDD4, CTC1 and DNAH11 taken forward from the segregation analysis, only NEDD4 exhibited a nominally significant asso-

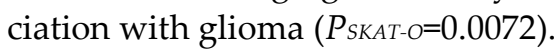

Table 2. Loss of heterozygosity (LOH) in gliomas with damaging NEDD4, CTC1, FLNA, DNAH11 variants. Evidence of allelic imbalance within a $1 \mathrm{Mb}$ window of given gene was used to assess potential LOH events. "LOH gain" - if significant gain of given variant allele in tumour relative to control, "LOH loss" - if significant loss of given variant allele relative to control.

\begin{tabular}{|c|c|c|c|c|c|}
\hline Gene & Protein change & No LOH & LOH gain & LOH loss & CNV \\
\hline NEDD4 & p.Met1162Thr & 1 & 0 & 0 & $15 q 21$ neutral \\
\hline NEDD4 & p.Pro1040Ala & 1 & 0 & 0 & $15 q 21$ neutral \\
\hline NEDD4 & p.Arg919Gln & 1 & 0 & 0 & $15 q 21$ neutral \\
\hline NEDD4 & p.Asn888Lys & 1 & 0 & 0 & $15 q 21$ neutral \\
\hline NEDD4 & p.Tyr873His & 1 & 0 & 0 & Chr15 loss \\
\hline NEDD4 & p.Glu242Val & 1 & 0 & 0 & $15 q 21$ neutral \\
\hline DNAH11 & p.Gly204Ser & 1 & 0 & 0 & Chr7 gain \\
\hline DNAH11 & p.Ala897Thr & 1 & 0 & 0 & Chr7 gain \\
\hline DNAH11 & p.Asp1165Gly & 1 & 0 & 0 & $7 p$ gain \\
\hline DNAH11 & p.His1166Pro & 0 & 0 & 1 & Chr7 gain \\
\hline DNAH11 & p.Gly2005Arg & 1 & 0 & 0 & Chr7 gain \\
\hline DNAH11 & p.Ile2418Ser & 1 & 0 & 0 & Chr7 gain \\
\hline DNAH11 & p.Gly2454Ala & 1 & 0 & 0 & Chr7 gain \\
\hline DNAH11 & p.Tyr3314Cys & 1 & 0 & 0 & Unknown \\
\hline DNAH11 & p.Glu3488Lys & 1 & 0 & 0 & Chr7 gain \\
\hline DNAH11 & p.Lys3593Asn & 1 & 0 & 0 & Chr7 gain \\
\hline DNAH11 & p.Lys3699Glu & 1 & 0 & 0 & Chr7 gain \\
\hline DNAH11 & p.Ile3705Val & 1 & 0 & 0 & 7p15 gain \\
\hline DNAH11 & p.Arg4111Gln & 1 & 0 & 0 & Unknown \\
\hline FLNA & p.Arg2073His & 1 & 0 & 0 & Xq28 neutral \\
\hline FLNA & p.Ala1141Thr & 1 & 0 & 0 & Xq28 neutral \\
\hline CTC1 & p.Val1111Ile & 1 & 0 & 0 & 17p13 neutral \\
\hline CTC1 & p.Val976Phe & 1 & 0 & 0 & 17p13 neutral \\
\hline CTC1 & p.Leu972Phe & 1 & 0 & 0 & 17p13 neutral \\
\hline CTC1 & p.Arg818Gln & 0 & 1 & 0 & 17p13 loss \\
\hline CTC1 & p.Pro813Ser & 0 & 1 & 0 & 17p13 neutral \\
\hline
\end{tabular}




\begin{tabular}{lcllll}
\hline CTC1 & p.Ser501Ile & 1 & 0 & 0 & $17 \mathrm{p} 13$ neutral \\
CTC1 & p.Ser340Trp & 0 & 1 & 0 & $17 \mathrm{p} 13$ neutral \\
CTC1 & p.Val288Met & 0 & 0 & 1 & $17 \mathrm{p} 13$ neutral \\
CTC1 & p.Ser234Ile & 1 & 0 & 0 & $17 \mathrm{p} 13$ neutral \\
\hline
\end{tabular}


Table 3. Damaging shelterin complex variants identified.Variant annotations are with respect to the canonical transcript ENST00000357628.3 and protein ENSP00000350249.3 for POT1, ENST00000276603.5 and ENSP00000276603.5 for TERF1, ENST00000603068.1 and ENSP00000474453.1 for TERF2, ENST00000300086.4 and ENSP00000300086.4 for TERF2IP, ENST00000267415 and ENSP00000267415.7 for TINF2 and ENST00000393919.4 and ENSP00000377496.4 for ACD.

\begin{tabular}{cccccccc}
\multicolumn{2}{c}{ ENSP00000300086.4 for TERF2IP, ENST00000267415 and ENSP00000267415.7 for TINF2 and ENST00000393919.4 and ENSP00000377496.4 for ACD. } \\
\hline Variant & Gene & Transcript change & Protein change & Consequence & N & Carrier & Histology \\
\hline 7:124493081_C/G & POT1 & c.814G $>$ C & p.Gly272Arg & Missense & 1 & TCGA-HW-7490 & Astrocytoma \\
7:124503601_G/A & POT1 & c.349C $>$ T & p.Arg117Cys & Missense & 1 & TCGA-FG-7637 & Astrocytoma \\
8:73921182_G/T & TERF1 & c.61G $>$ T & p.Ala21Ser & Missense & 1 & TCGA-DU-8165 & Oligodendroglioma \\
8:73926175_C/T & TERF1 & c.365C $>$ T & p.Thr122Met & Missense & 1 & TCGA-S9-A7IQ & Oligodendroglioma \\
16:69400867_T/C & TERF2 & c.1057A $>$ G & p.Lys353Glu & Missense & 1 & TCGA-DU-5854 & GBM \\
16:75688275_C/T & TERF2IP & c.775C $>$ T & p.Arg259Trp & Missense & 1 & TCGA-16-1045 & GBM \\
14:24709070_G/A & TINF2 & c.1289C $>$ T & p.Pro430Leu & Missense & 1 & TCGA-DB-A64R & Oligodendroglioma \\
16:67694222_G/A & ACD & c.160C $>$ T & p.Pro54Ser & Missense & 1 & FAM_75 & GBM \\
16:67693439_C/G & ACD & c.671+1G $>C$ & - & Splice acceptor & 1 & TCGA-27-2518 & GBM \\
\hline
\end{tabular}

7:124493081

7:124503601_G/A

GA-27-2518

GBM 


\section{Discussion}

To our knowledge, this study is one of the largest germline WES studies in glioma conducted to date. By prioritising genes containing variants segregating within families, our analysis implicates a number of candidates as susceptibility genes for glioma. Specifically, segregation analysis identified a number of candidate genes (i.e. FLNA, DNAH11, NEDD4, CTC1), which have not previously been implicated in glioma. While of potential interest gene-based tests failed to demonstrate a significant burden of variants in cases versus controls, implying further studies will be required to verify their importance in glioma.

FLNA (Filamin A) is an actin binding protein that regulates reorganisation of the actin cytoskeleton and therefore can affect cell shape and migration [28]. Localised mutations in FLNA have been shown to cause otopalatodigital syndrome types 1 and 2, frontometaphyseal dysplasia and Melnick-Needles syndrome [29]. While not previously linked with glioma, FLNA has been shown to interact with BRCA2 [30]. NEDD4 (Neural precursor cell expressed developmentally down-regulated protein 4) is an E3 ubiquitin ligase at 15q21 that plays a role in neural development and regulation of EGF signalling $[31,32]$. DNAH11 (Dynein heavy chain 11, axonemal) is a microtubule-dependent motor ATPase [33]. Homozygous or compound heterozygous variants in DNAH11 cause Ciliary Dyskinesia [34]. The carriers of DNAH11 p.Ser1470ArgfsTer6 in FAM_07 are however not known to show any symptoms of Ciliary Dyskinesia. Primary cilia are known to be important in neural stem cell differentiation [35], therefore presenting a plausible mechanism for DNAH11 mutations promoting glioma development through incomplete neural stem cell differentiation.

CTC1 (conserved telomere maintenance 1) is involved in protecting telomeres from degradation [36]. Mutations in CTC1 have been shown to cause Coats plus syndrome, a recessive pleiotropic disorder that can affect the brain [37]. Additionally, it has been demonstrated that CTC1 mutations can exert a dominant-negative effect on telomere maintenance and exhibit shorter telomeres [37, 38]. Intriguingly, tumour-normal analysis of 9 TCGA glioma samples carrying damaging CTC1 variants provided evidence for lossof-heterozygosity at 17p13 in 4 samples (Table 2). While consistent with CTC1 acting as a tumour suppressor gene the $17 \mathrm{p} 13$ region also contains the classic tumour-suppressor gene TP53, which may be driving the LOH.

Mutations in CTC1 are consistent with the emerging paradigm of telomere maintenance and glioma risk: notably common low-penetrance GWAS risk polymorphisms in TERT, RTEL, TERC [5,39] and STN1/OBFC1 [7] along with high-penetrance mutations in POT1 [11]. This study failed to identify any truncating mutations in POT1, however two missense variants predicted to be deleterious were observed as well as seven putatively damaging variants in other shelterin complex genes. Collectively, however mutations in the shelterin complex are unlikely to account for more than $2 \%$ of glioma cases (upper $95 \%$ confidence interval of point estimate).

Before a gene mutation can be considered as clinically actionable it requires rigorous validation and associated cancer risks to be established. The commentary on Bainbridge et al [11] emphasised that there was insufficient statistical support for POT1 being is a high-impact CSG and suggested conclusions overly reliant on inference from prior biology [40]. Although protein-truncating variants (PTVs) have a much higher probability of disease causation a priori, in the absence of experimental data it is difficult to assign functional effects per se. This is highlighted by a study making use of exome-sequence and transcriptomic data from the Genotype-Tissue Expression (GTEx) and Geuvadis projects, which has demonstrated shown that PTVs can exert tissue-specific effects [41]. Future studies in glioma may therefore make use of analyses of this kind to prioritise functionally relevant variants. 


\section{Conclusions}

In conclusion, this study describes exome sequencing of 168 glioma patients enriched for genetic susceptibility to glioma from 150 families to identify additional glioma predisposition genes. While a number of candidates are promising CSGs, our analysis provides little evidence to support the existence of high-impact PTVs contributing substantially to glioma risk comparable to $B R C A$ and breast cancer. This does preclude the existence of coding variants having moderate effects on glioma risk analogous to those in CHEK2, which have pleiotropic effects on cancer risk. Certainly, it is likely that POT1 variants may also have a similar risk profile.

Supplementary Materials: The following supporting information can be downloaded at: www.mdpi.com/xxx/s1, Figure S1: Principal components analysis (PCA) plots of the population ancestry of cases and controls; Table S1: Details of glioma cases sequenced; Table S2: Per-gene association analysis in glioma cases.

Author Contributions: Conceptualization, B.K. and R.H.; methodology, B.K., P.B., D.C. and R.H., formal analysis, B.K.; investigation, B.K., D.C., K.L., and A.D-B.; data curation, H.B. and O.C.; writing - original draft preparation, B.K. and R.H.; writing - review and editing, B.K., D.C., C.J., M.S. and R.H.; supervision, C.J., M.S. and R.H.; funding acquisition, M.S., C.J. and R.H. All authors have read and agreed to the published version of the manuscript.

Funding: This work was supported by grants from Cancer Research UK (C1298/A8362) and INCaDGOS-Inserm_12560 of the SiRIC CURAMUS. The Glioma International Case-Control Consortium Study was supported by grants from the National Institutes of Health, Bethesda, Maryland (R01CA139020, R01CA52689, P50097257, P30CA125123).

Institutional Review Board Statement: The study was conducted in accordance with the Declaration of Helsinki. The French part of the study was approved by the Ethics Committee of CPP-Ile de France VI (AC-2013-1962, 22/05/2014). The Danish part of the GLIOGENE project was accepted to be in accordance with Danish ethical regulations by the Danish Ethical Committee (VEK) system (File no. H-KF-2007-0027) and the Danish National Data Protection Board (File no. 2007-41-0138).

Informed Consent Statement: Informed consent was obtained from all subjects involved in the study.

Data Availability Statement: The whole exome data on the families and familial glioma cases generated and analysed during the current study are not publicly available due to restrictions imposed by respective ethics committees but researchers interested can contact the authors to gain access. The WES control data on 1958 Birth Cohort that supports this study have been deposited at the European Genome-phenome Archive (EGA), which is hosted by the European Bioinformatics Institute (EBI); accession numbers EGAD00001001021 and EGAS00001001667. The TCGA data is available from the database of Genotypes and Phenotypes (dbGaP), Study Accession: phs000178.v11.p8.

Acknowledgments: The results shown here are in whole or part based upon data generated by the TCGA Research Network: https://www.cancer.gov/tcga.

Conflicts of Interest: The authors declare no conflict of interest. The funders had no role in the design of the study; in the collection, analyses, or interpretation of data; in the writing of the manuscript, or in the decision to publish the results. 


\section{References}

1. Ostrom, Q.T.; H. Gittleman; J. Fulop; M. Liu; R. Blanda; C. Kromer; Y. Wolinsky; C. Kruchko; J.S. Barnholtz-Sloan, CBTRUS Statistical Report: Primary Brain and Central Nervous System Tumors Diagnosed in the United States in 2008-2012. Neuro Oncol, 2015. 17 Suppl 4: p. iv1-iv62.

2. Bondy, M.L.; M.E. Scheurer; B. Malmer; J.S. Barnholtz-Sloan; F.G. Davis; D. Il'yasova; C. Kruchko; B.J. McCarthy; P. Rajaraman; J.A. Schwartzbaum; et al., Brain tumor epidemiology: consensus from the Brain Tumor Epidemiology Consortium. Cancer, 2008. 113(7 Suppl): p. 1953-68.

3. Hodgson, S.V.; E.R. Maher; S. Hodgson, A practical guide to human cancer genetics. Vol. 2. 1999: Springer.

4. Hemminki, K.; S. Tretli; J. Sundquist; T.B. Johannesen; C. Granstrom, Familial risks in nervous-system tumours: a histologyspecific analysis from Sweden and Norway. Lancet Oncol, 2009. 10(5): p. 481-8.

5. Shete, S.; F.J. Hosking; L.B. Robertson; S.E. Dobbins; M. Sanson; B. Malmer; M. Simon; Y. Marie; B. Boisselier; J.Y. Delattre; et al., Genome-wide association study identifies five susceptibility loci for glioma. Nat Genet, 2009. 41(8): p. 899-904.

6. Kinnersley, B.; M. Labussiere; A. Holroyd; A.L. Di Stefano; P. Broderick; J. Vijayakrishnan; K. Mokhtari; J.Y. Delattre; K. Gousias; J. Schramm; et al., Genome-wide association study identifies multiple susceptibility loci for glioma. Nat Commun, 2015. 6: p. 8559.

7. Melin, B.S.; J.S. Barnholtz-Sloan; M.R. Wrensch; C. Johansen; D. Il'yasova; B. Kinnersley; Q.T. Ostrom; K. Labreche; Y. Chen; G. Armstrong; et al., Genome-wide association study of glioma subtypes identifies specific differences in genetic susceptibility to glioblastoma and non-glioblastoma tumors. Nat Genet, 2017. 49(5): p. 789-794.

8. Rajaraman, P.; B.S. Melin; Z. Wang; R. McKean-Cowdin; D.S. Michaud; S.S. Wang; M. Bondy; R. Houlston; R.B. Jenkins; M. Wrensch; et al., Genome-wide association study of glioma and meta-analysis. Hum Genet, 2012. 131(12): p. 1877-88.

9. Sanson, M.; F.J. Hosking; S. Shete; D. Zelenika; S.E. Dobbins; Y. Ma; V. Enciso-Mora; A. Idbaih; J.Y. Delattre; K. Hoang-Xuan; et al., Chromosome 7p11.2 (EGFR) variation influences glioma risk. Hum Mol Genet, 2011. 20(14): p. 2897-904.

10. Wrensch, M.; R.B. Jenkins; J.S. Chang; R.F. Yeh; Y. Xiao; P.A. Decker; K.V. Ballman; M. Berger; J.C. Buckner; S. Chang; et al., Variants in the CDKN2B and RTEL1 regions are associated with high-grade glioma susceptibility. Nat Genet, 2009. 41(8): p. 905-8.

11. Bainbridge, M.N.; G.N. Armstrong; M.M. Gramatges; A.A. Bertuch; S.N. Jhangiani; H. Doddapaneni; L. Lewis; J. Tombrello; S. Tsavachidis; Y. Liu; et al., Germline mutations in shelterin complex genes are associated with familial glioma. J Natl Cancer Inst, 2015. 107(1): p. 384.

12. Robles-Espinoza, C.D.; M. Harland; A.J. Ramsay; L.G. Aoude; V. Quesada; Z. Ding; K.A. Pooley; A.L. Pritchard; J.C. Tiffen; M. Petljak; et al., POT1 loss-of-function variants predispose to familial melanoma. Nat Genet, 2014. 46(5): p. 478-481.

13. Speedy, H.E.; B. Kinnersley; D. Chubb; P. Broderick; P.J. Law; K. Litchfield; S. Jayne; M.J.S. Dyer; C. Dearden; G.A. Follows; et al., Germ line mutations in shelterin complex genes are associated with familial chronic lymphocytic leukemia. Blood, 2016. 128(19): p. 2319-2326.

14. Palles, C.; J.B. Cazier; K.M. Howarth; E. Domingo; A.M. Jones; P. Broderick; Z. Kemp; S.L. Spain; E. Guarino; I. Salguero; et al., Germline mutations affecting the proofreading domains of POLE and POLD1 predispose to colorectal adenomas and carcinomas. Nat Genet, 2013. 45(2): p. 136-44.

15. Johanns, T.M.; C.A. Miller; I.G. Dorward; C. Tsien; E. Chang; A. Perry; R. Uppaluri; C. Ferguson; R.E. Schmidt; S. Dahiya; et al., Immunogenomics of Hypermutated Glioblastoma: A Patient with Germline POLE Deficiency Treated with Checkpoint Blockade Immunotherapy. Cancer Discov, 2016. 6(11): p. 1230-1236.

16. Legius, E.; L. Messiaen; P. Wolkenstein; P. Pancza; R.A. Avery; Y. Berman; J. Blakeley; D. Babovic-Vuksanovic; K.S. Cunha; R. Ferner; et al., Revised diagnostic criteria for neurofibromatosis type 1 and Legius syndrome: an international consensus recommendation. Genet Med, 2021. 23(8): p. 1506-1513.

17. Lunter, G.; M. Goodson, Stampy: a statistical algorithm for sensitive and fast mapping of Illumina sequence reads. Genome Res, 2011. 21(6): p. 936-9. 
18. Li, H.; R. Durbin, Fast and accurate short read alignment with Burrows-Wheeler transform. Bioinformatics, 2009. 25(14): p. 175460.

19. McKenna, A.; M. Hanna; E. Banks; A. Sivachenko; K. Cibulskis; A. Kernytsky; K. Garimella; D. Altshuler; S. Gabriel; M. Daly; et al., The Genome Analysis Toolkit: a MapReduce framework for analyzing next-generation DNA sequencing data. Genome Res, 2010. 20(9): p. 1297-303.

20. Van der Auwera, G.A.; M.O. Carneiro; C. Hartl; R. Poplin; G. Del Angel; A. Levy-Moonshine; T. Jordan; K. Shakir; D. Roazen; J. Thibault; et al., From FastQ data to high confidence variant calls: the Genome Analysis Toolkit best practices pipeline. Curr Protoc Bioinformatics, 2013. 11(1110): p. 1110 1-11 1033.

21. Power, C.; J. Elliott, Cohort profile: 1958 British birth cohort (National Child Development Study). Int J Epidemiol, 2006. 35(1): p. 34-41.

Flicek, P.; M.R. Amode; D. Barrell; K. Beal; K. Billis; S. Brent; D. Carvalho-Silva; P. Clapham; G. Coates; S. Fitzgerald; et al., Ensembl 2014. Nucleic Acids Res, 2014. 42(Database issue): p. D749-55.

23. Gonzalez-Perez, A.; N. Lopez-Bigas, Improving the assessment of the outcome of nonsynonymous SNVs with a consensus deleteriousness score, Condel. Am J Hum Genet, 2011. 88(4): p. 440-9.

24. Louis, D.N.; A. Perry; P. Wesseling; D.J. Brat; I.A. Cree; D. Figarella-Branger; C. Hawkins; H.K. Ng; S.M. Pfister; G. Reifenberger; et al., The 2021 WHO Classification of Tumors of the Central Nervous System: a summary. Neuro Oncol, 2021. 23(8): p. 1231-1251.

25. Ceccarelli, M.; F.P. Barthel; T.M. Malta; T.S. Sabedot; S.R. Salama; B.A. Murray; O. Morozova; Y. Newton; A. Radenbaugh; S.M. Pagnotta; et al., Molecular Profiling Reveals Biologically Discrete Subsets and Pathways of Progression in Diffuse Glioma. Cell, 2016. 164(3): p. 550-63.

26. Sathirapongsasuti, J.F.; H. Lee; B.A. Horst; G. Brunner; A.J. Cochran; S. Binder; J. Quackenbush; S.F. Nelson, Exome sequencing-based copy-number variation and loss of heterozygosity detection: ExomeCNV. Bioinformatics, 2011. 27(19): p. 2648-54. Lee, S.; M.J. Emond; M.J. Bamshad; K.C. Barnes; M.J. Rieder; D.A. Nickerson; N.G.E.S.P.-E.L.P. Team; D.C. Christiani; M.M. Wurfel; X. Lin, Optimal unified approach for rare-variant association testing with application to small-sample case-control wholeexome sequencing studies. Am J Hum Genet, 2012. 91(2): p. 224-37.

28. Ohta, Y.; J.H. Hartwig; T.P. Stossel, FilGAP, a Rho- and ROCK-regulated GAP for Rac binds filamin A to control actin remodelling. Nat Cell Biol, 2006. 8(8): p. 803-14.

29. Robertson, S.P.; S.R. Twigg; A.J. Sutherland-Smith; V. Biancalana; R.J. Gorlin; D. Horn; S.J. Kenwrick; C.A. Kim; E. Morava; R. Newbury-Ecob; et al., Localized mutations in the gene encoding the cytoskeletal protein filamin A cause diverse malformations in humans. Nat Genet, 2003. 33(4): p. 487-91.

30. Yuan, Y.; Z. Shen, Interaction with BRCA2 suggests a role for filamin-1 (hsFLNa) in DNA damage response. J Biol Chem, 2001. 276(51): p. 48318-24.

31. Lin, Q.; J. Wang; C. Childress; M. Sudol; D.J. Carey; W. Yang, HECT E3 ubiquitin ligase Nedd4-1 ubiquitinates ACK and regulates epidermal growth factor (EGF)-induced degradation of EGF receptor and ACK. Mol Cell Biol, 2010. 30(6): p. 1541-54.

32. Liu, Y.; R.W. Oppenheim; Y. Sugiura; W. Lin, Abnormal development of the neuromuscular junction in Nedd4-deficient mice. Dev Biol, 2009. 330(1): p. 153-66.

33. Vallee, R.B.; D. Varma; D.L. Dujardin, ZW10 Function in Mitotic Checkpoint Control, Dynein Targeting, and Membrane Trafficking: Is Dynein the Unifying Theme? Cell Cycle, 2014. 5(21): p. 2447-2451.

34. Knowles, M.R.; M.W. Leigh; J.L. Carson; S.D. Davis; S.D. Dell; T.W. Ferkol; K.N. Olivier; S.D. Sagel; M. Rosenfeld; K.A. Burns; et al., Mutations of DNAH11 in patients with primary ciliary dyskinesia with normal ciliary ultrastructure. Thorax, 2012. 67(5): p. 433-41.

35. Tong, C.K.; Y.G. Han; J.K. Shah; K. Obernier; C.D. Guinto; A. Alvarez-Buylla, Primary cilia are required in a unique subpopulation of neural progenitors. Proc Natl Acad Sci U S A, 2014. 111(34): p. 12438-43. 
36. Miyake, Y.; M. Nakamura; A. Nabetani; S. Shimamura; M. Tamura; S. Yonehara; M. Saito; F. Ishikawa, RPA-like mammalian Ctc1-Stn1-Ten1 complex binds to single-stranded DNA and protects telomeres independently of the Pot1 pathway. Mol Cell, 2009. 36(2): p. 193-206.

37. Anderson, B.H.; P.R. Kasher; J. Mayer; M. Szynkiewicz; E.M. Jenkinson; S.S. Bhaskar; J.E. Urquhart; S.B. Daly; J.E. Dickerson; J. O'Sullivan; et al., Mutations in CTC1, encoding conserved telomere maintenance component 1, cause Coats plus. Nat Genet, 2012. 44(3): p. 338-42.

38. Chen, L.Y.; J. Majerska; J. Lingner, Molecular basis of telomere syndrome caused by CTC1 mutations. Genes Dev, 2013. 27(19): p. 2099-108.

39. Walsh, K.M.; V. Codd; I.V. Smirnov; T. Rice; P.A. Decker; H.M. Hansen; T. Kollmeyer; M.L. Kosel; A.M. Molinaro; L.S. McCoy; et al., Variants near TERT and TERC influencing telomere length are associated with high-grade glioma risk. Nat Genet, 2014. 46(7): p. 731-5.

40. Cooke, J.G., RE: Germline Mutations in Shelterin Complex Genes Are Associated With Familial Glioma. J Natl Cancer Inst, 2015. 107(8).

41. Rivas, M.A.; M. Pirinen; D.F. Conrad; M. Lek; E.K. Tsang; K.J. Karczewski; J.B. Maller; K.R. Kukurba; D.S. DeLuca; M. Fromer; et al., Human genomics. Effect of predicted protein-truncating genetic variants on the human transcriptome. Science, 2015. 348(6235): p. 666-9. 